\title{
TRANSTORNOS DEPRESSIVOS: UM MATERIAL DIDÁTICO PARA EDUCAÇÃO A DISTÂNCIA ${ }^{1}$
}

\author{
Depressive disorders: didactic material for distance education
}

Trastornos depresivos: un material didáctico para educación a distancia

Mariluci Camargo Ferreira da Silva Candido²

Antonia Regina Ferreira Furegato ${ }^{3}$

\section{RESUMO}

A depressão é um grave problema de saúde pública freqüente nas unidades de atenção básica à saúde. A falta de conhecimento adequado do enfermeiro é um dos responsáveis pela deficiência na identificação e no cuidado aos deprimidos. Uma das respostas para transpor este impasse é a educação a distância. Nosso estudo teve por objetivo construir e analisar um material sobre depressão. 0 conteúdo teórico do material didático foi organizado em módulos. Os aspectos visuais e verbais do material foram testados por Especialistas com atuação no ensino e na assistência à depressão. Optou-se por uma amostra intencional. Utilizou-se um instrumento escalar tipo Likert. Os resultados apontaram que não houve discordância significativa quanto à opinião dos especialistas sobre o material produzido. Consideraram o conteúdo abrangente, atendendo aos objetivos instrucionais, com seqüência, exercícios e referências pertinentes.

Palavras-chave: Depressão. Educação a distância. Enfermagem.

\begin{abstract}
Depression is a frequent and severe public health problem in basic health care units. Nurses' lack of adequate knowledge is one of the factors responsible for the deficient identification and care delivery to depressed persons. One of the answers to overcome this deadlock is distance education. Our study aimed to construct and analyze material about depression. The theoretical content of the didactic material was organized in modules. The visual and verbal aspects of the material were tested by Specialists active in teaching and care delivery to depression. An intentional sample was chosen. A Likert-type instrument was used. The results indicated that there was no significant disagreement among the specialists' opinions on the produced material. They considered the content as comprehensive, complying with the instructional objectives through sequence, exercises and pertinent references.
\end{abstract}

\section{Resumen}

La depresión es un problema grave de salud pública frecuente en las unidades de atención básica a la salud. La falta de conocimiento adecuado del enfermero es uno de los responsables por la deficiencia en la identificación y en el cuidado a los deprimidos. Una de las respuestas para transponer este problema es la educación a distancia. La finalidad de nuestro estudio fue construir y analizar un material sobre depresión. El contenido teórico del material didáctico fue organizado en módulos. Los aspectos visuales y verbales del material fueron testados por Especialistas con actuación en la enseñanza y en la atención a la depresión. Se optó por una muestra intencional. Se utilizó un instrumento escalar tipo Likert. Los resultados apuntaron que no hubo discordancia significativa respecto a la opinión de los especialistas sobre el material producido. Consideraron el contenido abarcador, atendiendo a los objetivos instruccionales con secuencia, ejercicios y referencias pertinentes. 


\section{INTRODUCÃO}

A depressão é um transtorno mental considerado um grave problema de saúde pública. Estima-se que, no início deste século, 121 milhões de pessoas sofreram algum episódio depressivo durante a sua vida. É um dos transtornos mentais mais comuns, caracterizado por tristeza, perda de interesse em atividades cotidianas e diminuição da energia; é também um dos fatores mais prevalentes e potencialmente implicados nos mecanismos que conduzem à incapacidade e à utilização dos serviços de saúde ${ }^{1}$.

É um dos transtornos mentais que se apresenta com grande freqüência nas unidades de atenção básica à saúde. Muitas vezes não é reconhecida, e os pacientes só recebem tratamento para suas queixas físicas, passando despercebida a depressão. Esta falha em sua detecção estimula a cronificação e o sofrimento que poderiam ser evitados, se houvesse identificação precoce e encaminhamento adequado deste problema².

A falta de conhecimento atualizado do profissional de enfermagem tem sido citada como um dos fatores responsáveis pela deficiência na identificação e no cuidado de enfermagem aos pacientes deprimidos ${ }^{2,3}$.

Assim, tornam-se importantes a construção e a divulgação de estudos sobre o cuidado ao portador de transtorno depressivo os quais poderão nortear e sistematizar a conduta dos acadêmicos e dos enfermeiros diante do portador de transtorno depressivo.

Paralelamente, há que se considerar outro fator importante, vivemos em um país de dimensões continentais, e a missão de levar a formação adequada a todos os profissionais do Sistema Único de Saúde (SUS), pelos meios tradicionais, torna-se uma missão cara e de difícil realização. Uma das respostas para transpor este impasse é a Educação a Distância (EaD).

$\mathrm{A}$ EaD é um termo genérico que compreende uma série de estratégias usadas em educação por correspondência, universidades abertas, departamentos distantes de colégios ou universidades convencionais e treinamentos a distância de uma equipe de empresas privadas, mas também do estudo em casa ou no trabalho ${ }^{4}$. Entretanto, não deve ser vista como substitutiva de outras formas de educação convencional, mas como modalidade educacional alternativa.

Nesse sentido, o desenvolvimento de um material didático para educação a distância (EaD) sobre transtornos depressivos parece não só adequado, mas justificável por favorecer profissionais que se encontram longe dos centros acadêmicos, proporcionando oportunidade de informação e crescimento profissional.

Portanto, o objetivo deste estudo é apresentar o desenvolvimento e a análise de um material didático para educação a distância sobre transtornos depressivos.

\section{MATERIAL DIDÁTICO PARA EDUCAÇÃO A DISTÂNCIA}

No desenvolvimento de programas de EaD são identificados diversos problemas, entre eles a produção do material didático. Os sites das universidades exibem a fragilidade do seu material didático; via de regra, encontram-se simples tutoriais ou apostilas disponibilizadas eletronicamente, sugestões de leitura ou propostas de realização de exercícios preparatórios para provas 5 .

Na tentativa de minimizar a problemática da produção de materiais didáticos e cursos para EaD, é essencial que os educadores, ao idealizarem a criação de um curso a distância, conheçam e se orientem quanto ao planejamento e 0 desenvolvimento de um material capaz de provocar ou garantir a necessária interatividade do processo ensino-aprendizagem.

Um bom curso é mais do que conteúdo, também é pesquisa, é troca, é produção conjunta. Para suprir a menor necessidade presencial do professor, é importante ter materiais bem elaborados, auto-explicativos, com mais desdobramentos - links, textos de apoio, glossário e atividades ${ }^{6}$.

0 desenvolvimento de cursos virtuais deve contar com equipes interdisciplinares nas quais trabalhem docentes, tecnólogos em educação, especialistas em mídia, especialistas em testes, gráficos e redator. 0 curso é uma responsabilidade coletiva, amparado em princípios pedagógicos ${ }^{7,8}$.

Com as propostas metodológicas que utilizam o computador e a Internet, os usuários seguem algo pronto (tutorial), apóiamse em algo semidesenhado para complementá-los ou, até mesmo, criam algo diferente, sozinhos ou com os outros?.

Na preparação de curso a distância, consome-se mais tempo do que num curso tradicional. A conversão do material didático e o seu oferecimento requerem mais tempo e organização?.

Por se tratar de um aprendizado que pode ser feito a qualquer hora, cria-se a idéia de que tudo pode ser feito ao mesmo tempo. Para que isso não ocorra é recomendada a construção de um cronograma de aulas, com tópicos bem definidos e tarefas divididas por semanas. Para ajudar na compreensão do texto, devem-se colocar uma página com a tabela de conteúdos, o mesmo layout em todas as páginas, 0 texto da aula e a numeração de página no início; quebrar o texto quando a página tiver mais de três parágrafos e focar a atenção dos alunos em um tópico particular em cada aula ${ }^{10}$.

Tanto o curso presencial quanto o curso a distância de qualidade possuem os mesmos ingredientes. Ambos dependem de educadores maduros (intelectual e emocionalmente), entusiasmados e que saibam motivar e dialogar. Dependem, também, da curiosidade e da motivação dos alunos, o que facilita e estimula as melhores qualidades do professor. Também é necessário ter administradores, diretores e coordenadores mais abertos que entendam todas as dimensões do processo pedagógico em que estão envolvidos. Além disso, é necessário o devido apoio aos professores inovadores. Tanto o curso presencial como o curso a distância dependem de ambientes ricos de aprendizagem e adequada estrutura física (salas, tecnologias, bibliotecas). A aprendizagem não se faz só na 
sala de aula, mas nos inúmeros espaços de encontro, de pesquisa e produção que as grandes instituições propiciam aos seus professores e alunos ${ }^{6}$.

\section{METODOLOGIA DO ESTUDO}

\section{A - Desenvolvimento do material didático}

Para desenvolvimento do material didático foram reunidas informações sobre como os programas, cursos e unidades que utilizem recursos de EaD podem ser planejados e elaborados, segundo as diretrizes apresentadas por estudiosos de algumas universidades brasileiras ${ }^{11,12,13,14}$.

Foi feito o planejamento do programa do curso, partindose do levantamento de informações sobre a natureza do mesmo. 0 curso foi iniciado com a identificação do perfil dos alunos e objetivos da aprendizagem (gerais e específicos).

A clientela foi definida a partir da problemática da ausência ou ineficiência da assistência de enfermagem ao portador de depressão. 0 material didático foi dirigido para enfermeiros, principalmente os que trabalham nos serviços de atenção básica à saúde e que estão freqüentemente em contato com o portador de depressão.

Para o desenvolvimento, utilizou-se a abordagem instrucional. Nesta abordagem, o professor tem a função de criar situações que permitam a exposição do aluno ao conteúdo e privilegia a relação entre o professor e 0 aluno, no qual 0 papel principal é o do professor. É dividida em duas etapas: a elaboração do projeto e o desenvolvimento do curso ${ }^{11}$.

Para a elaboração do conteúdo de acordo com a abordagem instrucional, utilizaram-se todas as informações sobre transtornos depressivos que auxiliaram a organização do curso e forneceram aos alunos uma visão geral da proposta do curso (título, requisitos, público-alvo, objetivo geral, duração do curso, estratégias metodológicas, planejamento ou esboço do conteúdo).

A estrutura didática de um curso a distância é o produto de cerca de 12 a 18 meses de esforço com seu planejamento, desenvolvimento, experimentação e avaliação ${ }^{13}$.

Com base nas orientações de estudiosos deste tema ${ }^{12,13,14,15,16}$, procurou-se utilizar e adequar o estilo de escrita conversacional para a elaboração do material didático, no qual o professor fala com os alunos por meio da escrita, sendo amigável e incentivador, envolvendo-os em um diálogo. Para isso, utilizou um estilo pessoal, dirigindo-se aos alunos como "você" e referindo-se a si mesmo como "eu". Também, buscouse uma linguagem de fácil compreensão, considerando os seguintes aspectos: os parágrafos continham apenas uma idéia principal, ou duas idéias relacionadas; utilizaram-se frases curtas, contendo no máximo vinte palavras cada uma; evitaramse negações numa mesma frase; todos os termos técnicos foram explicados fazendo-se também a adequação da escrita à habilidade de leitura dos alunos.

0 conteúdo do material didático sobre transtornos depressivos foi organizado em nove módulos, levando-se em consideração o tema e a tecnologia ${ }^{1,3,12,13,15,16}$. 0 conteúdo programático foi dividido em:
1- Introdução: o que será que vem por aí? - apresentação dos participantes do curso e do ambiente on-line, importância do conhecimento sobre depressão para o enfermeiro e introdução a educação à distância

2- Conhecendo os aspectos históricos, conceituais, epidemiológicos e fatores de risco para a depressão

3- Classificação e o diagnóstico dos transtornos depressivos

4- Tratamento

5- Vamos falar das Psicoterapias? objetivos, tipos, vantagens e desvantagens

6- Farmacoterapia

7-Eletroconvulsoterapia e estimulação magnética transcraniana

8- Suicídio

9- Cuidado de enfermagem: atividades, relacionamento enfermeiro-cliente, processo de enfermagem.

Cada um desses módulos foi organizado de acordo com a seguinte estrutura: título do módulo, introdução ou apresentação com objetivos, apresentação do conteúdo a ser trabalhado e orientações para os alunos; desenvolvimento do conteúdo, glossário e destaques; bibliografia, leituras recomendadas e dicas de filmes.

Ao final de cada módulo, havia exercícios de caráter formal e obrigatório nos quais eram indicadas as leituras e as tarefas a serem executadas.

Para se estabelecer a carga horária do curso, foram englobadas todas as atividades do mesmo. Estabeleceram-se: 10 horas para leituras e estudo do conteúdo teórico distribuído em 1 hora por semana para leitura. A carga horária semanal foi assim distribuída: 1 para a leitura do conteúdo teórico, 1 para as tarefas, 1 para o chate 1 para o fórum de discussão. 0 total de horas de dedicação semanal ao curso deveria ser mais ou menos de 4 a 5 horas. 0 curso, composto por nove módulos com 4 horas semanais de atividades, perfazem 36 horas que, somadas às 4 horas para avaliação no final do curso, totalizam uma carga horária de 40 horas.

A avaliação programada para o curso é formativa, com atribuições de atividades em cada unidade: número de acessos e de postagens enviadas e qualidade das inferências durante as discussões nas ferramentas de comunicação (correio eletrônico, fórum de discussão e seções de bate-papo, etc); exercícios sob a coordenação e supervisão do professor/tutor; periodicidade de atualização de informações relevantes para o curso; uso da "netiqueta" (etiqueta de comportamento da Web). Ao final de cada módulo, há exercícios que devem ser realizados como condição indispensável para a obtenção do certificado de conclusão do curso.

0 texto do material didático desenvolvido na íntegra deve ser usado como material de apoio. Sua utilização em aulas virtuais requer a redução do conteúdo teórico para a apresentação das aulas. Do mesmo modo, é recomendado o uso de tópicos, figuras e interatividade uma vez que a comunicação é realizada por meio de palavras, símbolos e imagens. Nenhum curso de educação a distância estaria completo sem qualquer um destes recursos. Ilustrações, diagramas e outros meios audiovisuais são importantes em 
todo material educacional; são bons para a transmissão de idéias concretas, servindo de apoio ao ensino de conceitos e para a comunicação de idéias simultaneamente ${ }^{12}$.

\section{B- Análise do material didático por especialistas}

Para analisar este material didático, buscou-se determinar os elementos que deviam constituir o conteúdo sobre transtornos depressivos na educação a distância, obtendo informações úteis sobre o que deveria ou poderia ser acrescido ou retirado, de modo que os materiais elaborados pudessem corresponder aos objetivos para os quais foram desenvolvidos.

Alguns aspectos do material precisavam ser testados: 0 verbal e o visual. Ao examinar rapidamente uma unidade, o que é chamado de teste prévio dos elementos visuais, pode-se verificar um grande número de elementos além do conteúdo: sumário; títulos e subtítulos; seções suficientes e balanceadas; atividades (número e variedade); formato e disposição dos elementos; tipologia e corpo; comprimento; atratividade; paginação; numeração e ordenação. Diferentemente dos elementos visuais, os elementos verbais precisam ser lidos em detalhe para serem completamente verificados ${ }^{12}$.

Após a identificação do que deveria ser examinado, foi preciso saber e determinar de quem obter opiniões. Sabíamos que a obtenção de opiniões que proporcionam bom retorno sobre o assunto deve ser feita por diferentes grupos de pessoas que dominam 0 assunto, os especialistas.

Foi utilizada uma amostra intencional composta por 6 especialistas ( 4 enfermeiros, dos quais 2 atuam na assistência e 2 no ensino superior; 1 psicólogo que atua em psicologia clínica e 1 psiquiatra com atuação no ensino e na assistência). Optou-se por uma escala do tipo de Likert. A Escala do tipo Likert fornece ao pesquisador um instrumental para verificar 0 nível de concordância do sujeito por meio de graduações. Usualmente, tal escala contém 3, 5 ou 7 graduações a respeito das afirmativas que compõem uma questão a ser investigada.

No presente estudo, os noves módulos foram analisados por meio de sete assertivas nas quais os sujeitos foram convidados a pontuarem sua concordância, utilizando-se uma graduação de 0 a 5 pontos, no sentido crescente da concordância.

\section{RESULTADOS DA OPINIÃO DOS ESPECIALISTAS}

Primeiramente, discute-se a etapa de opinião sobre 0 material didático com base no instrumento formal.

Esperava-se que a escala utilizada pudesse dar indicadores mais expressivos e menos tendenciosos. Entretanto, a maioria dos sujeitos não apresentou discordância, ou seja, houve uma tendência dos participantes a optarem pelas respostas em concordância.
Quadro 1 - Respostas dos especialistas por nível de análise e concordância

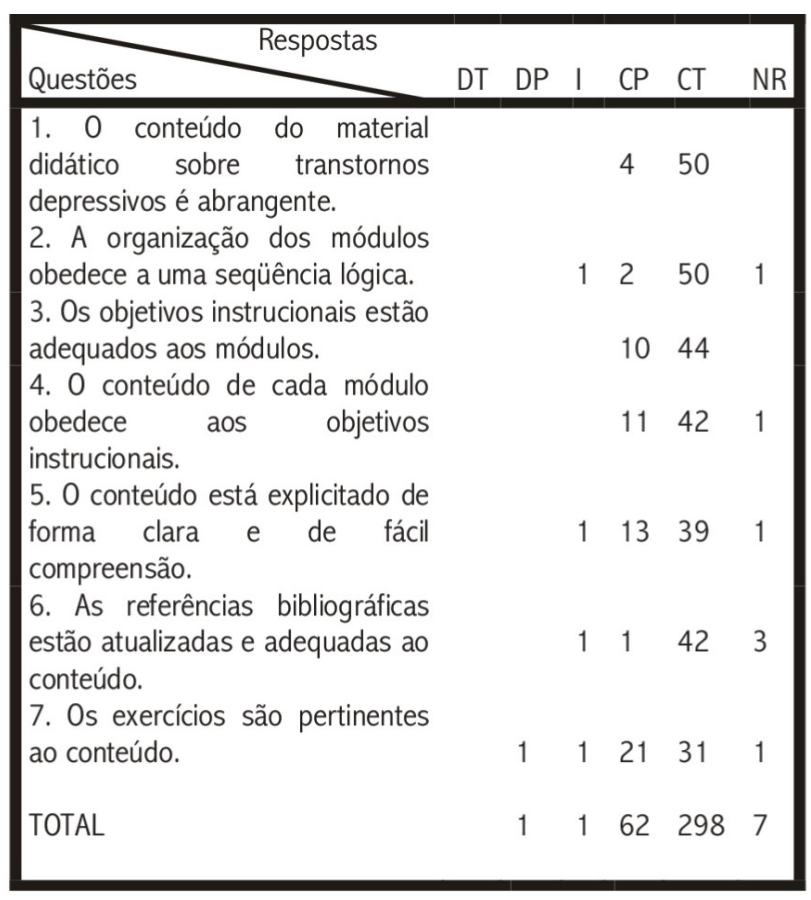

$\mathrm{DT}=$ discordo totalmente; $\mathrm{DP}=$ discordo parcialmente; $\mathrm{I}=$ =indiferente; $\mathrm{CP}=$ concordo parcialmente; $\mathrm{CT}=$ concordo totalmente; $\mathrm{NR}=$ não respondida

No Quadro 1 são apresentados os totais de respostas de todos os especialistas nos módulos, por nível de concordância.

A maioria das respostas ficou entre concordo parcialmente (62) e concordo totalmente (298), 4 assinalaram indiferente e 1 discordou parcialmente. Não houve respostas ao item discordo totalmente. Portanto, não houve indicações significativas de discordância.

Dentre as questões não respondidas (sete), observou-se que a maioria refere-se ao módulo nove. 0 fato de o tema do módulo ser específico da área de Enfermagem pode ter dificultado as respostas dos profissionais não-enfermeiros (médico e psicólogo).

Quanto à abrangência do conteúdo sobre transtornos depressivos do material didático (questão 1), as respostas foram: 50 para concordância total e 4 para concordância parcial. Observou-se uniformidade entre as respostas para todos os módulos, ficando entre 5 e 6 respostas por módulo. Portanto, pode-se dizer que o conteúdo do material didático foi abrangente.

Com relação à organização dos módulos em uma seqüência lógica (questão 2), as respostas foram: 50 para concordância total, 2 para concordância parcial e 1 para indiferente. Sendo assim, do total de 53 respostas, a maioria concordou com a organização seqüencial dos módulos.

Sobre a adequação dos objetivos instrucionais dos módulos (questão 3 e 4), 44 respostas foram de concordância total e 11 de concordância parcial. Da mesma forma, houve uniformidade em considerar que o conteúdo de cada módulo atende aos objetivos instrucionais, com 42 respostas para concordância total e 11 para concordância parcial. 
No que diz respeito ao conteúdo estar de forma clara e de fácil compreensão, houve 39 respostas para concordância total, 13 para concordância parcial e 1 para indiferente. Foi observado por um dos especialistas que alguns conteúdos se repetiam e deviam ser revistos.

Sobre a atualidade e adequação da referência bibliográfica e do conteúdo sobre transtornos depressivos (questão 6), 42 respostas foram para concordância total, 1 para concordância parcial e 1 para indiferente. A maioria das respostas apontou concordância indicando que as referências bibliográficas e 0 conteúdo foram considerados atualizados e adequados. Notamse algumas ausências de respostas para essa questão. Acreditase que os especialistas (não-enfermeiros) não tenham respondido por terem sido utilizadas algumas referências específicas da área de Enfermagem; não houve comentários ou recomendações a respeito do que faltaria para que o conteúdo do curso fosse considerado totalmente atualizado.

Quanto à pertinência dos exercícios ao conteúdo, 31 concordaram totalmente, 21 concordaram parcialmente, 1 foi indiferente, e houve 1 discordância parcial. Recomendou-se, para melhor fixação do conteúdo, a ampliação dos exercícios com discussão de casos.

Pelas respostas, pode-se dizer que o conteúdo do material didático foi considerado abrangente. A maioria concordou com a organização seqüencial dos módulos; houve uniformidade em considerar que o conteúdo de cada módulo atende aos objetivos instrucionais; não houve comentários ou observações sugerindo o que faltaria para que o conteúdo do curso fosse considerado totalmente atualizado, nem sobre a pertinência dos exercícios em relação ao conteúdo.

Após cada proposição, houve espaço para observações que o sujeito julgasse necessárias. Algumas recomendações foram feitas no texto do material didático deste curso por estes sujeitos, especialistas: uniformizar a seqüência de apresentação para todos os módulos e a apresentação de quadros com reflexões ou chamadas no texto; retirar a citação de autores no texto e usá-la somente na referência bibliográfica; ampliar exercícios com discussão de casos e focalizar a comunicação em temas relevantes como o relato da vida e do interesse do cliente. Cada um desses itens foi submetido à correção.

Melhorias no material didático podem ser rápidas e fáceis de se fazer, mas outras podem tomar mais tempo. Entre os itens mais facilmente modificados estão: correções de or tografia; correção dos erros de digitação; explicação de palavras difíceis; encurtamento de sentenças longas; numeração apropriada; correção de legendas e títulos de ilustrações; correção de erros de gramática e pontuação; dar as respostas certas para as perguntas e aperfeiçoamento dos títulos e subtítulos. Entre as modificações que levariam mais tempo para se fazer, incluemse: simplificar o conteúdo; adicionar novo conteúdo; reorganizar as seções da unidade; reescrever parágrafos; preparar uma nova tarefa ou teste de auto-avaliação e adicionar questões intratextuais ou novos objetivos ${ }^{12}$. Estes pontos foram considerados essenciais e foram todos revisados.

Todas as recomendações dos especialistas foram objeto de ponderação e revisão, sendo que as alterações no material didático foram incorporadas sempre que consideradas adequadas e pertinentes.

\section{CONSIDERACÕES FINAIS}

A elaboração e a análise do material didático sobre transtornos depressivos para EaD pretendem contribuir para a construção de outros materiais didáticos, especialmente na Enfermagem. A produção de material didático é um dos problemas mais importantes para o desenvolvimento de programas de $\mathrm{EaD}^{5}$.

Embora a interatividade seja destacada como um dos principais elementos na produção de material didático em EaD, este estudo se concentrou: na elaboração de materiais didáticos para educação a distância; nos procedimentos de planejamento do programa e do material didático mediado por computador.

0 processo de montagem do curso dentro do TelEduc é simples e se encontra disponível na EERP/USP, mas necessita de um administrador com conhecimentos de HTML e de servidores Web ou da assessoria do mesmo. Este curso deverá ser disponibilizado na plataforma TelEduc.

$\mathrm{Na}$ construção deste curso, observou-se a importância de uma equipe multiprofissional que trabalhe com questões relacionadas à didática, à pedagogia e à tecnologia de $\mathrm{EaD}, \mathrm{a}$ fim de viabilizar o desenvolvimento de projetos de EaD.

Com a experiência adquirida na realização deste curso, pode-se afirmar que o estudo e o desenvolvimento de materiais e cursos à distância são uma das possibilidades de pesquisa e com grande potencial e demanda para novos trabalhos.

De um modo geral, a elaboração e a análise deste curso foram satisfatórias. Os especialistas consideraram que 0 material didático atende ao objetivo de aperfeiçoar ou desenvolver as questões com que se defrontam os enfermeiros, acadêmicos de Enfermagem e outros profissionais da saúde, visando desenvolver estratégias mais eficientes nas ações voltadas ao indivíduo portador de transtorno depressivo, à sua família e à comunidade.

0 material produzido para EaD deverá atender a necessidade de formação e/ou atualização dos profissionais de Enfermagem, acadêmicos de Enfermagem e de outros profissionais de saúde no que se refere ao atendimento ao portador de transtorno depressivo, à sua família e à comunidade.

\section{Referências}

1.Willians A. Depression in the workplace: impact on employees. AAOHN J 1999; 47(11): 526-39.

2.Organización Panamericana de la Salud- OPS. Programa de salud mental. División de Promoción de Salud. Modelo para la capacitación de la enfermería general en al identificación y manejo de los trastornos afectivos. Washington(USA); 1999.

3.Silva MCF. Depressão: pontos de vista e conhecimento do enfermeiro da rede básica de saúde. [dissertação de mestrado] Ribeirão Preto(SP): Escola de Enfermagem / USP; 2001.

4.Keegan D. Foundations of distance education. London (UK): Routledge Taylor \& Francis; 1996.

5.Belisário A. 0 material didático na educação a distância e a constituição de propostas interativas. In: Silva M, organizadora. 
Educação on-line: teorias, práticas, legislação e formação corporativa. São Paulo (SP) Loyola; 2003.

6.Moran JM, Masetto MT, Behrens MA. Novas tecnologias e mediação pedagógica. Campinas (SP): Papirus; 2002.

7.Teixeira GJW, Bido DS, Spers EE, Giglio EM, Ohanian G, Siqueira JPL, Profera R. Capacitação de professores para o ensino a distância. Trabalho final da disciplina EAD 5824 - Didática II. São Paulo (SP): Faculdade de Economia, Administração e Contabilidade Universidade de São Paulo -. Departamento de Administração. Pós - Graduação em Administração. Doutoramento. DEDALUS - Acervo - FEA. São Paulo. $2^{\circ}$ Trimestre de 2000.

8.Gomez MV. Educação em rede: processo de criação de um curso web. [tese doutorado]. São Paulo (SP): Faculdade de Educação/USP; 2002.

9.Moran JM. Contribuições para uma pedagogia da educação on-line. In: Silva M. organizadora. Educação on-line: teorias, práticas, legislação e formação corporativa. São Paulo (SP): Loyola; 2003.

10.Knox ELS. The pedagogy os web site design. ALN Magazine [online]. 1997 Aug; [citado 7 nov 2007]; 1(2): Disponível em: http:// www.sloan-c.org/publications/magazine/v1n2/Knox.asp.
11.Universidade de Campinas-UNICAMP. Orientações para o desenvolvimento de cursos mediados por computador. [citado 7 nov 2007]. Disponível em: http://www.unicamp.br..

12.Laaser W, organizador. Manual de criação e elaboração de materiais para educação a distância. Tradução de Marcelo Carvalho de Oliveira. Brasília (DF): CEAD Ed/ UNB; 1997.

13.Peters 0. Didática do ensino a distância: experiências e estágio da discussão numa visão internacional. São Leopoldo(RS): Ed UNISINOS; 2001.

14.Amarante P. Saúde mental: políticas e instituições: programa de educação a distância. Rio de Janeiro(RJ): FIOTEC/FIOCRUZ-EAD; 2003. 15.Silva MRC. Aprendizagem. Bol EAD: Centro de Computação/UNICAMP [on-line] set 2001 [citado 7 nov 2007]. Disponível em: http:// www.ccuec.unicamp.br/ead/

16.World Health - WHO. Mental and neurological disorders. Dec 2001. [citado 7 nov 2007]. Fact sheet n.265. Disponível em: http:// www.who.int/mediacentre/factsheets/fs265/en/print.html.

17. Pasquali L. Instrumentos psicológicos. Manual prático de elaboração. Brasília (DF): IBAPP; 1999 